\title{
Effects of exercise on tests measuring attention and executive function in younger and older adults
}

Angela N. Burda, Jennifer Baldwin, Johanna Cravatta, Jody Heetland, Kayla Jacobs, Hannah Merfeld, Jaden Rausch, Mallory Rinnels, Regan Zollman

Department of Communication Sciences and Disorders, University of Northern lowa, IA, USA

Purpose: This study investigated whether or not performance differed between Young and Older Exercisers and between Young and Older Non-exercisers on the Attention Processing Training-Test (APT-Test), Behavioral Assessment of Dysexecutive Syndrome (BADS), and Functional Assessment of Verbal Reasoning and Executive Strategies (FAVRES).

Methods: A total of 119 participants were divided into the following groups: 46 Young Exercisers: (aged 18-40 years), 45 Older Exercisers (aged 60 and older), fourteen Young Nonexercisers, and fourteen Older Non-exercisers. Participants completed the APT-Test, BADS, and FAVRES in a counterbalanced manner. Independent sample t-tests were used to calculate outcomes.

Results: Young Exercisers had significantly higher scores than Older Exercisers on the APTTest, BADS Total Profile Score, and the FAVRES Total Accuracy Score. No significant differences occurred between Young and Older Exercisers on the FAVRES Total Rationale, Total Time, or Reasoning scores. No statistically significant differences occurred on any measures between Young and Older Non-exercisers.

Conclusions: Different performance trends occurred between the Exerciser groups and the Non-exerciser groups. For example, both Exerciser groups performed similarly on the FAVRES when asked to justify their responses and perform reasoning tasks. Older Exercisers provided correct responses for Accuracy measures, yet their responses did not earn the total points. No differences occurred on any test between the Non-exerciser groups. Thus, it could be helpful for clinicians to inquire about patients' pre-morbid exercise habits when using these tests. Participants were healthy, neurologically intact adults. Future research should investigate potential effects exercise has on these tests in adults with acquired neurological damage.

Keywords: Adult, Attention, Cognition, Executive function, Exercise

\section{INTRODUCTION}

Researchers have studied the effects of physical activity and measures of attention and executive function (EF) in adults across the lifespan. EF is a broad term that encompasses various complex cognitive processes used to analyze and assign meaning to incoming information, and these processes are often responsible for behaviors such as initiating, planning, organizing, sequencing, switching, inhibiting, and problem solving [1-7]. Having adequate EF allows individuals to competently function in daily life by assessing new incoming information, use prior knowledge from past experiences to ana-

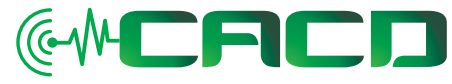

Received: December 19, 2020

Revision: April 15, 2021

Accepted: April 20, 2021

\section{Correspondence: \\ Angela N. Burda}

Department of Communication Sciences and Disorders, University of Northern lowa, 230 Communication Arts Center Cedar Falls, IA 50614-0356, USA

Tel: +1-319-273-3128

Fax: +1-319-273-6384 E-mail: angela.burda@uni.edu

C 2021 The Korean Association of SpeechLanguage Pathologists

This is an Open Access article distributed under the terms of the Creative Commons Attribution NonCommercial License (https://creativecommons.org/ licenses/by-nc/4.0/) which permits unrestricted noncommercial use, distribution, and reproduction in any medium, provided the original work is properly cited. 
lyze the current situation, determine a plan of action, and then reflect on the consequences $[3,5,7,8]$. EF disruptions can negatively impact decision making and self-control, leading to inappropriate behaviors and the inability to perform daily living skills. Thus, EF enables individuals to lead independent, fulfilling lives $[1,5,8,9]$.

Attention enables individuals to focus on relevant information and is comprised of several different types, including alternating attention in which individuals rapidly shift this focus amongst various stimuli, selective attention in which certain stimuli are focused on while others are not, and sustained attention in which target stimuli are focused on for an extended period of time $[1,7,10,11]$. Attentional skills allow persons to filter out irrelevant incoming information and focus on essential information to complete desired tasks $[1,7,10,12]$. EF and attention are posited to be interconnected and integrated cognitive mechanisms; the inability to focus our attention on relevant information leads to EF disruption $[1,4,10,12]$. Previous research studies have determined a relationship between aging and poor performance on cognitive measures [13-15]. Younan [16] suggests that age-related cognitive decline can result in decreased "processing speed, reasoning skills, memory, and executive functions." As individuals age, structures can display significant deterioration and undergo a reduction in neural connections that are necessary for advanced cognition skills like EF and attention. For instance, the hippocampus and frontal lobe experience a significant decline in functioning due to aging $[9,15]$. Aerobic exercise has been found to increase volume in the gray and white matter within the frontal and temporal lobes to preserve cognitive processes involved with attention and EF $[14,15,17]$. Therefore, exercise is a simple and inexpensive activity that could decrease the cognitive effects related to aging and positively impact performance on EF and attention tasks [17].

Many factors contribute to persons having robust cognitive skills during their lifetime, including the aforementioned engaging in exercise $[16,18,19]$. For example, Hawkins, Kramer, and Capaldi found that older adult exercisers had significantly better performance on dual attention tasks than older adult non-exercisers [20]. Yaffee et al. [21] reported that participants aged 65 and older who exercised had less risk of having cognitive declines, and that the more older adults exercised, the less likely they were to develop cognitive declines later in life. Eggemont et al. [18] had participants complete an activity questionnaire as well as various $\mathrm{EF}$ and cognitive tests. Older adult participants who were more physically active, including those who exercised, had higher scores on all EF and cognitive measures versus older adults who were not as physically active [18]. More recently, young adults in a high physical activity group, which included exercise, had significantly higher EF measures versus young adults in a low physical activity/exercise group [22]. Lower levels of physical activity and exercise in 4,555 adults aged 50 and older followed for six years correlated with lower verbal fluency and letter cancellation scores; participants with higher physical activity levels also had better EF [23]. Thus, persons across the lifespan who exercise can have higher scores on attention and EF measures.

There are many factors to consider when discussing the broad term of "exercise." "Exercise" can often be synonymous with "physical activity" [24,25], yet researchers have attempted to define differences between the terms. Being engaged in physical activities can be subjective, and vary across individuals and at various points in their lives [24], however, the type of physical activity, the intensity of physical activity, and the duration of physical activity can have different impacts on cognition [25]. Household tasks (e.g., yardwork, cleaning, home repair) can be considered physical activities, some of which can require significant exertion. Tools such as the Physical Activity Scale for the Elderly (PASE) can be used to determine older adults' physical activity by asking questions regarding their participation in activities such as sitting (e.g., reading, watching TV), walking, light sport or recreational activities (e.g., bowling, fishing), moderate sport or recreational activities (e.g., golfing without a golf cart, ice skating), and strenuous sport or recreational activities (e.g., jogging, swimming) [26]. Exercise has been defined as "physical activity that is planned, structured, repetitive, and purposive in the sense that improvement or maintenance of one or more components of physical fitness is an objective" [24]. McKinney et al. [27]. defined exercisers as individuals whose primary goals are to be healthy and fit and who exercise more than 2.5 hours of the week. Previous research studies investigating cognition have developed various criteria for both the inclusion of participants and separating participants into different groups based on the above noted factors (i.e., type, intensity, and duration of exercise) [28]. Erickson et al. [15] randomly assigned participants into either an aerobic or a stretching and toning exercising group, while Colcombe et al. had all participants attend 1-hour exercise programs, three days a week [14].

Tests used in the current study have been described in previous studies [13,29-31]. The Attention Process Training Test (APT-Test) evaluates simple sustained, complex sustained, se- 
lective, divided, and alternating attention [32]. Researchers read aloud the instructions and played a $\mathrm{CD}$ for each of the five subtests (i.e., levels) in which numbers are read aloud; levels increased in difficulty. Persons press a clicker when they hear a correct response (e.g., a number that is one less than the number immediately prior to it during the complex sustained attention subtest). For level 1, the simple sustained attention subtest, individuals press the clicker when they hear the number "two" read aloud. As previously described, participants click when they hear a number that is one less than the number right before it for Level 2, the complex sustained subtest. Participants press the clicker when they hear the number "two" while random words are being said aloud in the background during level 3, the selective attention subtest. For the divided attention task (level 4), participants click when they hear the number "two" while simultaneously using a pen or pencil to cross off even numbers (e.g., 2, 4, 6) in a display of numbers on a $81 / 2$ " $\times 11^{\prime \prime}$ piece of paper. For level 5 , alternating attention and the last task, persons click when they hear a number that is one less than the number right before it. When the researcher says "switch" during the audio presentation, participants then have to click when hearing a number that is one more than the number immediately prior to it. Switching events occur numerous times. The test manual states that administration takes approximately 20-30 minutes, and reports normative data for an original sample of 25 control individuals $\left(\mathrm{M}_{\text {age }}=26.3\right)$ and 25 persons with mild brain injuries $\left(\mathrm{M}_{\text {age }}=\right.$ 27.2). Means and standard deviations for the age groups of 18 29 years, $30-39$ years, $40-59$ years, and $60-85$ years old are also reported [32].

Both EF tests used in the current study have also been described by researchers [13,29,31]. The Behavioural Assessment of the Dysexecutive Syndrome (BADS) [33] includes six tests (i.e., subtests) to assess $\mathrm{EF}$ abilities. Verbal, written, and hands-on responses are required. In the first test, persons quickly learn one rule pertaining to a set of playing cards (e.g., hearts, spades), perform the target task, quickly unlearn the original rule and learn a new rule, then complete the task using the new rule. Examples of other tests participants complete include responding to temporal judgement questions (e.g., How long does it take to blow up a party balloon?), mapping out paths to zoo exhibits per specific rules, and drawing how they would look for lost keys in a field. The examiners manual reports the test can be given in 40-50 minutes and was normed on 216 healthy adults divided into four age groups: 16-31, 32-47, 48-63, 64 years and older, as well as on
78 individuals (aged 19-78) with neurological disorders [33].

For the Functional Assessment of Verbal Reasoning and Executive Strategies (FAVRES) [34], participants are administered four subtests and provide verbal and written responses. Subtests include, but are not limited to: finding a suitable birthday activity for a young male relative based on a list of available activities, budget constraints, and schedule. Another subtest requires individuals to schedule a workday when given a list of tasks that need to be done, some of which are higher priority than others. Participants also complete prediction and generation tasks for each subtest (e.g., what good and bad things could happen from meeting with an angry customer; generate as many ways to advertise as possible). The FAVRES takes generally 50-60 minutes to give and was normed on 101 healthy persons aged 17-89 years and 52 adults with acquired brain injury $[34,35]$. Both the BADS and FAVRES are ecologically valid tests speech-language pathologists (SLPs) could give their adult neurogenic patients $[35,36]$.

The APT-Test [32], BADS [33], and FAVRES [34] have been used across several research studies to measure EF and attention $[3,8,13,29-31,35,37-40]$. Johnston recently conducted a survey that included 51 practicing speech-language pathologists (SLPs) who developed a list of formalized assessments administered to evaluate their adult patients' cognition [41]. The relative frequency of usage of the APT-Test [32], BADS [33], and FAVRES [34] was estimated based on responses in the survey. The FAVRES [34] was listed by $32 \%$ percent of SLPs as a formalized assessment commonly administered to their patients and was rated as the second most popular assessment used to evaluate cognition [41]. The APT-Test [32] and BADS [33] were also listed in the top 20 cognitive assessments used by practicing SLPs. Other studies investigating the effects of physical activity or exercise on aspects of cognition have used differing cognitive tests [18,42], such as the Trailmaking Test (TMT) [43], the Hopkins Verbal Learning Test-Revised (HVLT-R) [44], and the Clock-in-a-Box Test (CIB) [45]. However, based on the data gathered from Johnston's [41] survey, such tests are not used by clinicians working in the field [41]. Rather, the APT-Test [32], BADS [33], and FAVRES [34] are common formalized assessments administered by practicing SLPs [41].

Despite such research, more information is needed. The lack of research on the effects of exercise on attention and EF tests that SLPs may give their adult patients makes it essential to further explore any potential relationship. In addition, no studies have been found in which attention and EF test scores 
on these specific assessments have been compared between older and younger adult exercisers and older and younger adult non-exercisers, respectively. Determining if exercise has an effect on these tests' scores contributes to the literature base and may add to the information SLPs would want to obtain from their patients and/or patients' family members and caregivers. The following research questions were addressed: 1) Are there performance differences on the APT-Test [32], the BADS [33], and the FAVRES [34] between younger and older adult Exercisers? 2) Are there performance differences on the APT-Test [32], the BADS [33], and the FAVRES [34] between younger and older adult Non-Exercisers?

\section{METHODS}

\section{Participants}

The participants for this cross sectional quasi-experimental study were approved by the University of Northern Iowa's Institutional Review Board (Protocol \# 19-0125). Participants were recruited from small, mid-sized, and large metropolitan communities and from rural areas in the Midwest by posting flyers in public areas (e.g., community centers). Similar to prior studies $[13,29,30]$, inclusion criteria included: no neurological impairment or disorders; be a native English speaker; minimum of a high school education; score of 28 or higher on the Mini-Mental State Examination (MMSE) [46]. Upon meeting inclusion criteria, participants were asked to report whether or not they exercised. Those who indicated they did were classified as Exercisers, which were specifically defined as individuals who engaged in various physical activities a minimum of three times per week in order to improve or maintain their fitness and health [27]. Exercisers were asked to report on average, how frequently during the week they exercised, and what kinds of physical activities they partook in. For the purpose of this study, acceptable physical activities included walking, swimming, running, jogging, biking, lifting weights, attending a workout class, and/or doing yoga [23,
27,47]; housework and yard work were not considered "exercise" [24]. Participants who reported they did not exercise were categorized as Non-exercisers. A power analysis for an effect size of .08 with an alpha of .05 indicated that a minimum of 105 participants was necessary. A total of 119 adults participated and were broken down into the following groups: 46 Young Exercisers: (aged 18-40 years), 45 Older Exercisers (aged 60 and older), fourteen Young Non-exercisers, and fourteen Older Non-exercisers. Age groups were defined based on prior studies [13,29]. Table 1 includes mean ages and mean MMSE scores for the four participant groups.

\section{Stimuli, Procedures, and Data Analysis}

Once individuals met all inclusion criteria and responded to the questions about exercising, participants completed an individual session in which the APT-Test [32], BADS [33], and FAVRES [34] were administered in a counterbalanced order. Length of sessions generally ranged from 90-120 minutes with breaks provided. Although hearing screenings were not conducted [35], researchers presented directions at a comfortable volume level and adjusted their volume, if necessary.

Data analysis was similar to prior studies $[3,8,13,29,31,48]$. Overall total test scores for the BADS and FAVRES were used for analyses. For the APT-Test [32], no overall test score was calculated. Thus, Residual Scores (i.e., total responses correct) were reported for each of the five APT-Test [32] subtests: sustained, complex sustained, selective, divided and alternating. The possible maximum score was 24 for the alternating attention subtest; the total score possible for the other four subtests was 30. For the BADS [33], an overall Total Profile Score ranging from 0-24 was obtained by adding up the Profile Scores from each of the six tests (i.e., subtests). For the FAVRES [34], participants obtained the maximum points possible if they provided responses containing the most pieces of correct information; some points were given if they provided responses that were sufficient but lacked all of the necessary pieces. Raw scores were converted into Total Standard Scores for Accu-

Table 1. Mean Age and MMSE Scores

\begin{tabular}{|c|c|c|c|c|c|c|c|c|}
\hline & \multicolumn{2}{|c|}{$\begin{array}{l}\text { Young exercisers } \\
\qquad(n=46)\end{array}$} & \multicolumn{2}{|c|}{$\begin{array}{l}\text { Older exercisers } \\
\qquad(n=45)\end{array}$} & \multicolumn{2}{|c|}{$\begin{array}{l}\text { Young non-exercisers } \\
\qquad(n=14)\end{array}$} & \multicolumn{2}{|c|}{$\begin{array}{l}\text { Older non-exercisers } \\
\qquad(n=14)\end{array}$} \\
\hline & $M$ & $S D$ & $M$ & $S D$ & $M$ & $S D$ & $M$ & $S D$ \\
\hline Age & 23.57 & 3.69 & 69.87 & 7.81 & 24.43 & 4.48 & 65.93 & 6.38 \\
\hline MMSE* & 29.83 & 0.64 & 29.11 & 0.91 & 29.71 & 0.47 & 29.50 & 0.76 \\
\hline
\end{tabular}

MMSE, mini-mental state examination.

${ }^{*}$ The highest possible score is 30 . 
racy, Rationale, Time, and Reasoning $(M=100, S D=15)$. As noted by Kelkar, Hough, and Fang, higher Accuracy, Rationale and Reasoning scores indicated better participant performance; higher scores on Timing indicate poorer performance (i.e., target task took longer) [48].

\section{Reliability}

Pearson $r$ correlations were calculated using raw scores on $20 \%$ of a randomly chosen sample (i.e., 24 participants). The researchers and a trained graduate speech-language pathology student's scores were correlated for inter-rater reliability. The researchers scored the protocols twice for intra-rater reliability; the second scoring took place four weeks following the initial scoring. Inter-rater reliability for the APT was $r=0.90$; intra-rater reliability was $r=0.92$. Inter-rater reliability for the BADS was $r=0.88$; intra-rater reliability was $r=0.94$. Interrater reliability for the FAVRES was $r=0.89$; intra-rater reliability was $r=0.95$.

\section{RESULTS}

\section{Descriptive statistics}

Overall mean scores were obtained for each test (Table 2). As previously noted, residual scores for each subtest on the APTTest were calculated since no overall total score is obtained. The Total Profile Score was obtained for the BADS, and Total Accuracy, Total Rationale, Total Timing, and Reasoning Standard Scores were calculated for the FAVRES. Older Exercisers had lower mean scores on all three tests compared to Younger
Exercisers. Older Non-exercisers had slightly higher scores on the APT-Test's Sustained Attention subtest and higher mean scores on the FAVRES' Total Accuracy score versus Young Non-exercisers.

\section{Inferential statistics}

Independent sample t-tests were conducted to determine if statistically significant differences occurred between Young and Older Exercisers and between Young and Older Non-exercisers.

\section{Exercisers}

Statistically significant differences occurred between Young and Older Exercisers on all APT-Test subtests: Sustained Attention $(t(89) 1.39, p \leq 0.007)$, Complex Sustained Attention $(t(89)=$ $4.51, p \leq 0.0001)$, Selective Attention $(t(89)=4.01, p \leq 0.0001)$, Divided Attention $(t(89)=4.87, p \leq 0.0001)$, and Alternating Attention $(t(89)=4.77, p \leq 0.003)$. Young Exercisers also had statistically higher scores on the BADS Total Profile Score $(t(89)=$ $3.45, p \leq 0.015)$ and FAVRES Total Accuracy score $(t(89)=4.88$, $p \leq 0.0001)$ versus Older Exercisers. No statistically significant differences occurred between the two groups on the following FAVRES scores: Total Rationale $(t(89)=2.18, p>0.39)$, Total Time $(t(89)=2.20, p>0.48)$, and Reasoning $(t(89)=5.15, p>0.30)$ (Table 3$)$.

\section{Non-Exercisers}

No statistically significant differences occurred between Younger and Older Non-exercisers on any of the three tests. More specifi-

Table 2. Mean APT-Test, BADS, and FAVRES Scores

\begin{tabular}{|c|c|c|c|c|c|c|c|c|c|c|}
\hline \multirow[t]{2}{*}{ Tests } & \multirow{2}{*}{$\begin{array}{l}\text { Subtest or } \\
\text { score name }\end{array}$} & \multirow{2}{*}{$\begin{array}{c}\text { Total } \\
\text { score } \\
\text { possible }\end{array}$} & \multicolumn{2}{|c|}{$\begin{array}{l}\text { Young exercisers } \\
\qquad(n=46)\end{array}$} & \multicolumn{2}{|c|}{$\begin{array}{l}\text { Older exercisers } \\
\quad(n=45)\end{array}$} & \multicolumn{2}{|c|}{$\begin{array}{l}\text { Younger non-exercisers } \\
\qquad(n=14)\end{array}$} & \multicolumn{2}{|c|}{$\begin{array}{l}\text { Older non-exercisers } \\
\qquad(n=14)\end{array}$} \\
\hline & & & $M$ & $S D$ & $M$ & $S D$ & $M$ & $S D$ & $M$ & $S D$ \\
\hline \multirow[t]{5}{*}{ APT-Test } & Sustained & 30 & 29.98 & 0.15 & 29.84 & 0.64 & 29.14 & 2.66 & 29.93 & 0.27 \\
\hline & Complex sustained & 30 & 25.91 & 4.03 & 20.40 & 7.22 & 26.14 & 4.82 & 21.07 & 4.41 \\
\hline & Selective & 30 & 26.50 & 3.14 & 21.64 & 7.58 & 27.00 & 4.54 & 21.43 & 6.15 \\
\hline & Divided & 30 & 28.52 & 1.31 & 25.73 & 3.65 & 28.29 & 1.77 & 27.00 & 2.63 \\
\hline & Alternating & 24 & 20.50 & 3.26 & 15.78 & 5.85 & 19.86 & 5.16 & 16.43 & 3.59 \\
\hline BADS & Total profile score & 24 & 18.78 & 2.03 & 16.91 & 3.05 & 18.71 & 2.27 & 17.50 & 2.50 \\
\hline \multirow[t]{4}{*}{ FAVRES } & Total SS Accuracy & 111 & 93.11 & 15.39 & 69.62 & 28.67 & 81.36 & 17.72 & 94.21 & 14.50 \\
\hline & Rationale & 111 & 83.72 & 21.58 & 72.67 & 26.67 & 87.43 & 17.18 & 81.14 & 21.62 \\
\hline & Time & 126 & 109.89 & 13.16 & 103.55 & 14.16 & 113.00 & 12.04 & 100.07 & 13.30 \\
\hline & Reasoning & 142 & 99.76 & 16.12 & 79.31 & 21.42 & 93.93 & 11.90 & 81.93 & 11.54 \\
\hline
\end{tabular}

SS, standard score. 
Table 3. Exercisers' Independent Samples t-test Results for APT-Test, BADS, and FAVRES

\begin{tabular}{lccc}
\hline Tests & $t$ & $d f$ & $p$-value \\
\hline APT-Test sustained attention & 1.39 & 89 & 0.007 \\
APT-Test complex sustained attention & 4.51 & 89 & 0.0001 \\
APT-Test selective attention & 4.01 & 89 & 0.0001 \\
APT-Test divided attention & 4.87 & 89 & 0.0001 \\
APT-Test alternating attention & 4.77 & 89 & 0.003 \\
BADS total profile score & 3.45 & 89 & 0.015 \\
FAVRES total accuracy & 4.88 & 89 & 0.0001 \\
FAVRES total rationale & 2.18 & 89 & $0.39^{*}$ \\
FAVRES total time & 2.20 & 89 & $0.48^{*}$ \\
FAVRES reasoning & 5.15 & 89 & $0.30^{*}$
\end{tabular}

*Results were not statistically significant.

cally, no statistically significant differences occurred on any of the APT-Test subtests: Sustained Attention $(t(26)=-1.10$, $p>0.06)$, Complex Sustained Attention $(t(26)=2.91, p>0.59)$, Selective Attention $(t(26)=2.73, p>0.23)$, Divided Attention $(t(26)=1.52, p>0.17)$, and Alternating Attention $(t(26)=2.04$, $p>0.49$ ). Also, no statistically significant differences occurred between both Non-exerciser groups on the BADS Total Profile Score $(t(26)=1.35, p>0.42)$ or on the following FAVRES scores: Total Accuracy $(t(26)=-2.10, p>0.63)$, Total Rationale $(t(26)=$ $0.85, p>0.57)$, Total Time $(t(26)=2.70, p>0.50)$, and Reasoning $(t(26)=2.71, p>0.44)($ Table 4$)$.

\section{DISCUSSION}

This study sought to determine if performance differences occurred on the APT-Test [32], the BADS [33], and the FAVRES [34] between Young and Older Exercisers and between Young and Older Non-exercisers, respectively. Young Exercisers had significantly higher scores versus Older Exercisers on the APTTest, the BADS Total Profile Score, and the FAVRES Total Accuracy Score. No significant differences occurred between Young and Older Exercisers on the FAVRES Total Rationale, Total Time, or Reasoning scores. No statistically significant differences occurred on any measures between the Young Non-exercisers and Older Non-exercisers. Younger adults having higher scores than older adults on the APT-Test, BADS, and FAVRES has been documented $[13,38]$. In addition, data indicate that in general, older adults can have poorer performance on attention and executive function scores versus younger adults $[14,15]$. Researchers have also reported that
Table 4. Non-Exercisers' Independent Samples t-test Results for APT-Test, BADS, and FAVRES*

\begin{tabular}{lccc}
\hline Tests & $t$ & $d f$ & $p$-value \\
\hline APT-Test sustained attention & -1.10 & 26 & 0.06 \\
APT-Test complex sustained attention & 2.91 & 26 & 0.59 \\
APT-Test selective attention & 2.73 & 26 & 0.23 \\
APT-Test divided attention & 1.52 & 26 & 0.17 \\
APT-Test alternating attention & 2.04 & 26 & 0.49 \\
BADS total profile score & 1.35 & 26 & 0.42 \\
FAVRES total accuracy & -2.10 & 26 & 0.63 \\
FAVRES total rationale & 0.85 & 26 & 0.57 \\
FAVRES total time & 2.70 & 26 & 0.50 \\
FAVRES reasoning & 2.71 & 26 & 0.44
\end{tabular}

${ }^{*}$ No statistically significant differences occurred between Young and Older Non-Exercisers for any tests.

physically active adults have higher attention and executive function scores than those who are not as physically active $[18,20-23]$. This is the first known study to compare the performance on tests SLPs may use between younger and older adults who exercise as well as between younger and older adults who do not exercise.

As noted, although Young Exercisers and Older Exercisers had differences on several scores (i.e., APT-Test, BADS Total Profile Score, FAVRES Accuracy), they did not evidence any statistically significant difference on the FAVRES' Rationale, Time, or Reasoning Scores. This suggests that overall, the two groups of exercisers performed generally similarly when asked to provide justifications for the responses they chose, that they took a similar amount of time to complete the various tasks, and they were able to adequately perform generating and predicting tasks. Tasks on the FAVRES [34] much more closely resemble that of activities found in everyday life. Persons do not necessarily press a clicker when listening for target numbers in a string of numbers with changing rules, such as those found in the APT-Test [32], nor do they tend to complete worksheets denoting which paths they would take when searching for keys or visiting exhibits, or verbally respond to quickly changing rules regarding playing cards, such as those tasks found in the BADS [33]. However, similar to tasks in the FAVRES [34], most individuals must plan their day, prioritize what is important and must be dealt with sooner rather than later, and manage when unexpected circumstances arise. Persons may also find themselves at some point in time needing to complain or have concerns about a repair not being done correctly. Thus, tasks in this test more 
closely resemble that of everyday life and are more ecologically valid in nature [34].

While there were statistically significant differences on the FAVRES' Total Accuracy score, Older Exercisers still generally provided accurate responses. The issue is that Older Exerciser participants did not provide responses in which they earned the highest points possible for Accuracy. This trend was reported in a prior study in which South Asian adults choose correct responses, but also did not provide responses in which they earned the highest points compared to White adult participants [31]. Thus, Older Exercisers' responses were not necessarily incorrect per se, but rather lacked all of the target information that would have led to higher scores. As noted in Kallambettu, Burda, and Wakeman, instructions given to the participants do not state that choosing or providing specific responses will lead to participants earning the highest points possible [31]. It is possible that the Older Exercisers would have had higher scores had more explicit test directions denoted that more pieces of correct information would have led to higher scores. Nonetheless, Older Exercisers were just as successful as Young Exercisers when providing rationales for their chosen answer and when asked to provide generating and predictive responses (i.e., Reasoning scores). This is not necessarily surprising as older adults can have equally robust problem solving and reasoning skills as younger adults, particularly for scenarios that are more daily life oriented [49-51]. However, researchers are not necessarily in agreement if older adults do well in more ecologically-based problem solving research tasks simply because they have more years of real world experience [50]. Prior studies have also found that older adults generally take longer on the FAVRES' than younger adults [13,38], yet no Time differences occurred in the current study. Older adults can be efficient problem solvers [51], and it is conceivable the ecologically valid tasks on the FAVRES may have minimized any potential performance differences between the two differently-aged Exerciser groups [52].

It is interesting no differences occurred between Young Exercisers and Older Non-exercisers. This was somewhat unexpected as prior studies noted above have shown performance differences on measures of attention or executive function with respect to age $[13,15,38]$ and physical activity $[18,20,22$, 23]. However, Younan recently investigated potential performance differences in EF scores in young-old adult exercisers, old-old adult exercisers, young-old sedentary adults, and oldold sedentary adults [16]. While young-old adults (aged 4567) consistently scored better in digit span and latency tasks than old-old adults (aged 72-94) regardless of activity level, the old-old adult exercisers scored worse on latency measures than old-old sedentary adults. No significant differences occurred amongst the different groups on the Wisconsin cardsorting task [16]. Although younger adults were not part of the Younan study, results from that study indicate that it is possible there may be no performance differences on certain cognitive measures, regardless if individuals exercise [16]. SLPs may not typically ask patients about exercise habits when using the APT-Test [32], BADS [33], or FAVRES [34], however, the current study suggests it is plausible such inquiries should be made to better gain an overall understanding of patients' premorbid functioning.

\section{Limitations and future research}

There are several limitations with this study. First, participants self-reported whether or not they exercised. Although other researchers have obtained participant self reports on exercise $[18,21,22]$, objective data has also been obtained (e.g., accelerometer; number of steps per day) $[19,22,47]$. Also, older adults' self-reports of physical activity may differ from their actual physical activity [49]. The current study asked participants to report whether or not they exercised and to report on the amount; they were not asked to report the intensity of exercise (e.g., light, moderate), yet older adults' exercise may differ in its intensity compared to younger adults [47]. In addition, participants were not asked to document their overall physical activity. Some household activities and yardwork could be similar to exercise (e.g., washing windows, splitting and stacking wood). Participants may not have been considered formal exercisers, yet were quite physically active. Therefore, the activities the researchers considered "exercise" may have not truly captured the degree of physical fitness that participants possessed. Another limitation is that the study only took into account whether or not participants were exercising at the time of the study. Other variables were not included as part of the data analysis, such as whether or not participants smoked [53] or if they were involved in some kind of socially engaging activities either through work or volunteering [54, 55]. Their verbal and non-verbal intelligence abilities were not tested, yet all of these factors have been found to have implications on adults' cognitive functioning $[56,57]$.

Age group classifications were based on prior research studies [13,30,58]. However, age groupings have been variable in prior studies relating to aging, cognition, and also exercise $[15,16,22,23,38,59]$. Only three participants were in their 80 s, 
none were in their 90s; the majority of Older Exercisers and Older Non-exercisers participants were in their 60s. Yet, some researchers have reported that real world problem solving may decline in adults over the age of 75 [60]. It is possible that including more individuals in their 80s and 90s, perhaps even as a stand alone group, would have yielded different results. In addition, all participants were neurologically intact. It is difficult to fully understand possible clinical implications exercise may have on individuals' scores on the tests used in this study until acquired neurological damage is present.

Future research should include replicating this study using objective measures in addition to self reports. Including measures of intensity and/or questionnaires, such as the Physical Activity Scale for the Elderly (PASE) [61] or Paffenberger Physical Activity Questionnaire [62] could also be beneficial. In addition, it would be helpful to include participant groups representing more diverse age groups. This study consisted of healthy adult participants. However, obtaining data on the premorbid exercise habits of persons with acquired neurogenic communication disorders could inform SLPs if asking about exercise habits is of particular clinical value when administering any of the tests used in this study.

\section{ACKNOWLEDGMENTS}

This study was presented November 2019 at the Annual Meeting of the American Speech-Language-Hearing Association in Orlando, Florida.

\section{REFERENCES}

1. Diamond A. Executive functions. Annual Review of Psychology. 2013;64:135-168.

2. Georgiou GK, Das JP. Direct and indirect effects of executive function on reading comprehension in young adults. Journal of Research in Reading. 2018;41:243-258.

3. Higginson CI, Thompson T, Benjamin AE, Rosales AC, Zane KL, Valenti M, et al. Construct validity of the Functional Assessment of Verbal Reasoning and Executive Strategies (FAVRES). Brain Injury. 2017;31:1807-1812.

4. Miyake A, Friedman NP, Emerson MJ, Witzki AH, Howerter A, Wager TD. The unity and diversity of executive functions and their contributions to complex "frontal lobe" tasks: a latent variable analysis. Cognitive Psychology. 2000;41:49-100.

5. Murray L. Focusing attention on executive functioning in Aphasia. Aphasiology. 2017;31:721-724.

6. Pickens S, Ostwald SK, Murphy-Pace K, Bergstrom N. Evidence synthesis: Systematic review of current executive function mea- sures in adults with and without cognitive impairments. International Journal of Evidence-Based Healthcare. 2010;8:110-125.

7. Yogev-Seligmann G, Hausdorff JM, Giladi N. The role of executive function and attention in gait. Movement Disorders. 2008;23:329342.

8. Fukuta D, Mori C. Intervention program to improve executive functions and enhance planning abilities of patients with mild neurocognitive disorder. Rehabilitation Nursing. 2019;44:263-270.

9. Jurado MB, Rosselli M. The elusive nature of executive functions: A review of our current understanding. Neuropsychology Review. 2007;17:213-233.

10. Gordon-Pershey M, Wadams A. The relationship of language and attention in elders with nonfluent aphasia. Cogent Medicine. 2017;4:1-18.

11. VanSolkema M, McCann C, Barker-Collo S, Foster A. Attention and communication following TBI: Making the connection through a meta-narrative systematic review. Neuropsychology Review. 2020;30:345-361.

12. Mak C, Whittingham K, Cunnington R, Boyd RN. Efficacy of mindfulness-based interventions for attention and executive function in children and adolescents: A systematic review. Mindfulness. 2018;9:59-78.

13. Burda A, Anderson E, Berryman M, Davis C, Heun M, Kise T. Performance of young, middle-aged, and older adults on tests of executive function. Canadian Journal of Speech-Language Pathology and Audiology. 2017;41:277-286.

14. Colcombe SJ, Erickson KI, Scalf PE, Kim JS, Prakash R, McAuley E, et al. Aerobic exercise training increases brain volume in aging humans. The Journals of Gerontology Series A. 2006;61:11661170 .

15. Erickson KI, Voss MW, Prakash RS, Basak C, Szabo A, Chaddock L, et al. Exercise training increases size of hippocampus and improves memory. Proceedings of the National Academy of Sciences. 2011;108:3017-3022.

16. Younan B. Cognitive functioning differences between physically active and sedentary older adults. Journal of Alzheimer's Disease Reports. 2018;2:93-101.

17. Churchill JD, Galvez R, Colcombe S, Swain R, Kramer AF, Greenough WT. Exercise, experience, and the aging brain. Neurobiology of Aging. 2002;3:941-955.

18. Eggemont LH, Milberg WP, Lipsitz LA, Scherder EJ, Leveille SG. Physical activity and executive function in aging: The MOBILIZE Boston study. Journal of the American Geriatrics Society. 2009;57: 1750-1756.

19. Voss MW, Weng TB, Burzynska AZ, Wong CN, Cooke GE, Clark R, et al. Fitness, but not physical activity, is related to functional integrity of brain networks associated with aging. Neuroimage. 2016;131:113-125.

20. Hawkins HL, Kramer AF, Capaldi D. Aging, exercise, and attention. Psychology and Aging. 1992;7:643-653.

21. Yaffee K, Barnes D, Nevitt M, Lui L, Covinsky K. A prospective study of physical activity and cognitive decline in elderly women. Archives of International Medicine. 2001;161:1703-1708. 
22. Matsuda K, Ikeda S, Mitsutake T, Nakahara M, Nagai Y, Ikeda T, et al. Factors influencing executive function by physical activity level among young adults: A near-infrared spectroscopy study. Journal of Physical Therapy Science. 2017;29:470-475.

23. Daly M, McMinn D, Allan JL. A bidirectional relationship between physical activity and executive function in older adults. The Frontiers in Human Neuroscience. 2015;8:1044.

24. Caspersen CJ, Powell KE, Christenson GM. Physical activity, exercise, and physical fitness: Definitions and distinctions for healthrelated research. Public Health Reports. 1985;100:126-131.

25. Norton K, Norton L, Sadgrove D. Position statement on physical activity and exercise intensity terminology. Journal of Science and Medicine in Sport. 2010;13:465-502.

26. Logan SL, Gottlieb BH, Maitland SB, Meegan D, Spriet LL. The Physical Activity Scale for the Elderly (PASE) questionnaire: Does it predict physical health? International Journal of Environmental Research and Public Health. 2013;10:3967-3986.

27. McKinney J, Velghe J, Fee J, Isserow S, Drezner J. Defining athletes and exercisers. The American Journal of Cardiology. 2018;123: 532-535.

28. Tsuk S, Netz Y, Dunsky A, Zeev A, Carasso R, Dwolatzky T, et al. The acute effect of exercise on executive function and attention: Resistance versus aerobic exercise. Advances in Cognitive Psychology. 2019;15:208-215.

29. Burda AN, Gilbert JL, Amundson C, Baughman K, Brummel A, Crimmins S, et al. Do scores on an attention test predict scores on executive function tests? Clinical Archives of Communication Disorders. 2018;3:192-200.

30. Burda A, Baker K, Burns A, Butler C, Clark L, Cook W, et al. Performance of young and middle-aged adults on cognitive tests. Journal of Medical Speech-Language-Pathology. 2014;21:393-404.

31. Kallambettu V, Burda A, Wakeman N. South Asian adults' performance on executive function tests. American Journal of SpeechLanguage Pathology. 2017;26:1254-1261.

32. Sohlberg MM, Mateer CA. APT-Test: Attention Process TrainingTest. Lash \& Associates, 2005.

33. Wilson BA, Alderman N, Burgess PW, Emslie HC, Evans JJ. BADS: Behavioral Assessment of Dysexecutive Syndrome. United Kingdom: Thames Valley, 1996.

34. MacDonald S. FAVRES: Functional Assessment of Verbal Reasoning and Executive Strategies. Ontario: CCD Publishing, 2005.

35. MacDonald S, Johnson CJ. Assessment of subtle cognitive communication deficits following acquired brain injury: A normative study of the Functional Assessment of Verbal Reasoning and Executive Strategies (FAVRES). Brain Injury. 2005;19:895-902.

36. Norris G, Tate RL. The Behavioural Assessment of the Dysexecutive Syndrome (BADS): Ecological, concurrent and construct validity. Neuropsychology Rehabilitation. 2000;10:33-45.

37. Bartfai A, Markovic G, Landahl KS, Schult ML. The protocol and design of randomized controlled study on training of attention within the first year after acquired brain injury. BMC Neurology. 2014;14:1-9.

38. Marcotte K, McSween MP, Pouliot M, Martineau S, Pauze A,
Wiseman-Hakes C, et al. Normative study of the Functional Assessment of Verbal Reasoning and Executive Strategies (FAVRES) test in the French-Canadian population. Journal of Speech, Language, and Hearing Research. 2017;60:2217-2227.

39. Markovic G, Schult ML, Elg M, Bartfai A. Beneficial effects of early attention process training after acquired brain injury: A randomized controlled trial. Journal of Rehabilitation Medicine. 2020;52: $1-9$.

40. Preston J, Ballinger C, Gallagher H. Understanding the lived experience of people with multiple sclerosis and dysexecutive syndrome. British Journal of Occupational Therapy. 2014;77:484- 490.

41. Johnston E. The role of the speech language pathologist in concussion management: A survey analysis. Master's Thesis. University of Oregon, 2018.

42. Carlson MC, Saczynski JS, Rebok GW, Seeman T, Glass TA, McGill $\mathrm{S}$, et al. Exploring the effects of an "everyday" activity program on executive function and memory in older adults. The Gerontologist. 2008;48:793-801.

43. Lezak MD, Howieson DB, Bigler ED, Tranel D. Neuropsychological assessment. 5th ed. New York: Oxford University Press, 2012.

44. Brandt J, Benedict RH. Hopkins Verbal Learning Test Revised (HVLT). Lutz, FL: Psychological Assessment Resources, 1991.

45. Grande L, Milberg W, Rudolph J, Gaziano M, McGlinchey R. A timely screening for executive functions and memory. Journal of International Neuropsychology Society. 2005;11:9-10.

46. Folstein MF, Folstein SE, Fanjiang G. Mini-Mental State Examination: Clinical guide. Lutz, FL: Psychological Assessment Resources, 2001.

47. Herbolsheimer F, Riepe MW, Peter R. Cognitive function and the agreement between self-reported and accelerometer-accessed physical activity. BMC Geriatrics. 2018;18:1-9.

48. Kelkar AS, Hough M, Fang A. Do we think alike? A cross-cultural study of executive functioning. Culture and Brain. 2013;1:118-137.

49. Berg CA. Everyday problem solving in context. In: Hofer SM, Alwin DF, editors. Handbook of cognitive aging: Interdisciplinary perspectives. California: Sage Publications Inc, 2008. p. 207-223.

50. Berg CA, Meegan SP, Klaczynski P. Age and experiential differences in strategy generation and information requests for solving everyday problems. International Journal of Behavior Development. 1999;23:615-639.

51. Blanchard-Fields F, Chen Y, Norris L. Everyday problem solving across the adult life span: Influence of domain specificity and cognitive appraisal. Psychology and Aging. 1997;12:684-693.

52. Crawford S, Channon S. Dissociation between performance on abstract tests of executive function and problem solving in reallife type situations in normal aging. Aging \& Mental Health. 2002; 6:12-21.

53. Amini R, Sahli M, Ganai S. Cigarette smoking and cognitive function among older adults living in the community. Aging, Neuropsychology, and Cognition. 2021;28:616-631.

54. Pugh E, De Vito A, Divers R, Robinson A, Weitzner DS, Calamia M. Social factors that predict cognitive decline in older African American adults. International Journal of Geriatric Psychiatry. 2020;36: 
403-410.

55. Williams KN, Kemper S. Interventions to reduce cognitive decline in aging. Journal of Psychosocial Nursing and Mental Health Services. 2010;48:42-51.

56. DeYoung CG, Quilty LC, Peterson JB, Gray JR. Openness to experience, intellect, and cognitive ability. Journal of Personality Assessment. 2014;96:46-52.

57. Ramsden S, Richardson FM, Josse G, Thompas MS, Ellis C, Shakeshaft $\mathrm{C}$, et al. Verbal and non-verbal intelligence changes in the teenage brain. Nature. 2011;479:113-116.

58. Burda AN, Casey AM, Foster TR, Pilkington AK, Reppe EA. Effects of accent and age on transcription of medically related utterances: A pilot study. Communication Disorders Quarterly. 2006;27:110116.
59. Kvavilashvili L, Kornbrot DE, Mash V, Cockburn J, Milne A. Differential effects of age on prospective and retrospective memory tasks in young, young-old, and old-old adults. Memory. 2009;17: 180-196.

60. Gilhooly ML, Gilhooly KJ, Phillips LH, Harvey D, Brady A, Hanlon P. Real-world problem solving and quality of life in older people. British Journal of Health Psychology. 2007;12:587-600.

61. Washburn RA, McAuley E, Katula J, Mihalko SL, Boileau, RA. The Physical Activity Scale for the Elderly (PASE): Development and evaluation. Journal of Clinical Epidemiology. 1993;46:153-162.

62. Paffenberger RS, Blair SN, Lee IM, Hyde RT. Measurement of physical activity to assess health effects in free-living populations. Medicine \& Science in Sports \& Exercise. 1993;25:60-70. 